Citation: Hirt C. and Seeber G. (2008) Accuracy Analysis of vertical deflection data observed with the Hannover Digital Zenith Camera System TZK2-D. Journal of Geodesy 82(6): 347-356. DOI: 10.1007/s00190-007-0184-7. 


\title{
Accuracy analysis of vertical deflection data observed with the Hannover Digital Zenith Camera System TZK2-D
}

Received: date / Accepted: date

\begin{abstract}
This paper analyses the accuracy of vertical deflection measurements carried out with the Digital Zenith Camera System TZK2-D, an astrogeodetic state-of-the-art instrumentation developed at the University of Hannover. During 107 nights over a period of 3.5 years, the system was used for repeated vertical deflection observations at a selected station in Hannover. The acquired data set consists of about 27300 single measurements and covers 276 hours of observation time, respectively. For the data collected at an earlier stage of development (2003 to 2004), the accuracy of the nightly mean values has been found to be about $0.10-0$ !'12. Due to applying a refined observation strategy since 2005, the accuracy of the vertical deflection measurements was enhanced into the unprecedented range of $0 . \prime 05-0 . .08$. Accessing the accuracy level of $0 . .05$ requires usually 1 hour of observational data, while the 0. '08 accuracy level is attained after 20
\end{abstract}

C. Hirt

Institut für Erdmessung, Leipniz Universität Hannover, Schneiderberg 50, 30167 Hannover, Germany

Present Address:

C. Hirt

Department of Geomatics, HafenCity University Hamburg, Hebebrandstr. 1, 22297 Hamburg, Germany

E-mail: Chris.Hirt@web.de

G. Seeber

Institut für Erdmessung, Leipniz Universität Hannover, Schneiderberg 50, 30167 Hannover, Germany

E-mail: Guenter@ursmaris.de 
minutes measurement time. In comparison to the analogue era of geodetic astronomy, the accuracy of vertical deflection observations is significantly improved by about one order of magnitude.

Keywords Digital Zenith Camera System (DZCS) · vertical deflection · accuracy

\section{Introduction}

During the last years considerable advancements were made in geodetic astronomy with the development of Digital Zenith Camera Systems (DZCS) in Hannover and Zurich (Hirt and Bürki 2002; Bürki et al. 2004; Hirt 2004). These transportable and completely automated instruments are employed at field stations in order to determine the direction of the plumb line and, by taking geodetic coordinates into account, the deflection of the vertical.

With respect to conventional visual and photographic instrumentation from the analogue era of geodetic astronomy, the performance of the DZCS is significantly improved due to using chargecoupled device (CCD) technology for star imaging and applying the new high-precision UCAC and Tycho-2 star catalogues as celestial reference. Observation and processing of the digital star images is completely automated, accelerating significantly the acquisition of vertical deflection data.

Several years ago the determination of vertical deflections often required $2-3$ hours or even more, using conventional astrogeodetic instrumentation such as analogue zenith cameras (e.g. Wissel 1982, Bürki 1989) or astrolabes. Nowadays, the observation and processing of vertical deflection data at single stations takes a total of about 20 minutes. Depending on the season (duration of darkness) and the distance between the stations, the new DZCS allow to collect vertical deflection data at $10-20$ stations per night. In recent time, this improvement in efficiency lead to an increasing application of DZCS in gravity field determination at local scales (Hirt 2004; Hirt and Seeber 2006; Hirt et al. 2006; Hirt and Flury 2007) and at regional scales (Müller et al. 2004; Brockmann et al. 2004; Müller et al. 2006).

Besides the enhanced efficiency, the new DZCS provide vertical deflection data more accurate than conventional astrogeodetic instruments which were mostly operated at the $0 . \prime 3-0.5$ accuracy level (e.g. Wissel 1982; Bürki 1989). First studies on the accuracy of the new DZCS showed that vertical deflections are determined accurate to 0.10 - 0"'15 (e.g. Hirt et al. 2004, Hirt 2004). This accuracy range estimate was based on repeated observations at selected stations in Northern Germany (e.g. data collected during 14 nights at Hannover station), residuals analysed between double 
occupations of several stations in different nights and based on independent comparison data. Comparisons were made between the astrogeodetic observations and vertical deflections derived from the gravimetric geoid model EGG97, and from photographic zenith tube observations, however, without detecting significant differences. Furthermore, a site located in Switzerland (Geostation Zimmerwald) was observed both with the Zurich and Hannover DZCS over 5 and 7 nights, respectively, yielding an agreement at the $0 . \prime 1$ accuracy level (Müller et al. 2004).

Since the publication of the first studies on the DZCS accuracy, the observational precision of the Hannover DZCS could be further improved due to the development of a refined observation technique (cf. Sect. 2). Since 2005, the observation series at the Hannover station has been considerably extended with observations carried out over a total of 107 different nights. This new data set is the most comprehensive one acquired at a single station with a DZCS to date. The Hannover DZCS vertical deflection observations provide statistically well founded accuracy estimates, as over a total time span of 3.5 years - a wide spectrum of environmental conditions and resulting refraction is covered by the data. These aspects motivate a new analysis of the attainable accuracy of vertical deflections observed with a DZCS.

This paper is organized as follows. Sect. 2 briefly introduces the DZCS instrumentation used for this study as well as the observation techniques applied. The vertical deflection observations used in this study are described in Sect. 3 and the data processing is briefly outlined in Sect. 4. An analysis of accuracy with focus on the error sources affecting the observations and on the relation between the number of observations and the attainable accuracy is presented in Sect. 5. Eventually, some concluding remarks are given in Sect. 6.

\section{Instrumentation}

The instrumentation used in this study is the Digital Zenith Camera System TZK2-D (Transportable Zenitkamera 2 - Digitalsystem), developed and operated at the Institut für Erdmessung of the University of Hannover. The DZCS consists of a zenith camera equipped with a CCD imaging sensor, which is used for the determination of astronomical latitude and longitude $(\Phi, \Lambda)$. The second component is a GPS receiver which is used for time tagging of the exposure epochs as well as for determining geodetic latitude and longitude $(\varphi, \lambda)$ of the camera. By combining both components vertical deflections

$\xi=\Phi-\varphi$ 
$\eta=(\Lambda-\lambda) \cos \varphi$

at the surface are obtained, which are also known as Helmert vertical deflections (e.g. Heiskanen and Moritz 1967, Torge 2001). Vertical deflections $(\xi, \eta)$ are the measure for the slope of the equipotential surface with respect to the ellipsoid the geodetic coordinates $(\varphi, \lambda)$ refer to. They play an important role in astrogeodetic gravity field determination (e.g. Heiskanen and Moritz 1967, Marti 1997, Torge 2001, Hirt and Flury 2007).

In sequence, a short description of the measurement concepts and recently realized improvements is given. Details on the technical realization of the Hannover DZCS TZK2-D with particular focus on the use of CCD technology for digital star imaging and the use of GPS for time tagging of the exposure epochs are given by Hirt and Bürki (2002), Hirt (2004) and Hirt (2006).

In order to refer the DZCS observations to the direction of the plumb line, tilt measurements are performed before and during star imaging. A pair of high-precision tilt meters, installed in perpendicular orientation onto the DZCS, permanently read the actual inclination of the camera with respect to the plumb line. Depending on the inclination values, motorized mechanics (three motor cylinders) and a computer control the levelling of the camera. The alignment of the camera to the plumb line is automatically accomplished to an accuracy of a few arc seconds. Along with the observation process, small but inevitably occurring deviations between the principal camera axis and the plumb line (e.g. due to sinking of the instrument) are monitored by the tilt meters and corrected for.

The DZCS is featured by a motorized bearing which enables the camera body to be rotated in two directions, differing by $180^{\circ}$ in azimuth. Observing in both camera directions eliminates the influence of zero point errors of the tilt meters as well as of the CCD. As a refinement, observations are generally performed in a time-symmetric sequence (directions $1-2-2-1$ ), so that also linear variations of the zero point errors are cancelled out.

Another instrumental error source with systematic behaviour is the azimuth-dependent error of the DZCS, which may reach amplitudes of a few arc seconds and vary in the range from some 0 ".01 to a few 0 ". 1 . (for details refer to Hirt 2004). In order to reduce this systematic effect, a calibration technique is applied where at each station the observation series in two directions are performed in three or four different azimuthal zenith camera system orientations. In early 2005, efforts were made to overcome the azimuth dependency of DZCS observations by applying a more sophisticated observation technique. Herein the complete camera body, including its tripod, is rotated between opposite directions by means of an additional motorized precision bearing. 
The decided advantage of this approach over the calibration technique is a much more effective reduction of the azimuth error and its variations, leading to a significantly smaller instrumental noise level. For the analysis of vertical deflection data in the following sections, it is useful to introduce two different levels of performance of the Hannover DZCS.

Level of performance 1: Up to 2004, the instrumental set-up (levelling, focusing) of the DZCS as well as the rotation between both camera directions required human intervention whereas the data acquisition was already completely automated. At all stations, the calibration technique was applied in order to reduce the azimuth-dependent camera error.

Level of performance 2: Since an extensive reworking of the instrumentation in early 2005, the instrumental set-up as well as the camera rotation are accomplished by means of the installed motor set. As a consequence, a completely automated observation of vertical deflections is achieved and any influences attributable to the operator are avoided. The above mentioned additional precision bearing is used for the rigorous elimination of the azimuth-dependent error.

\section{Observations}

After instrumental set-up, which usually takes a few minutes, single observations of vertical deflections are automatically performed. A single observation comprises two images of zenithal stars, exposed in opposite camera directions, as well as the exposure epochs and tilt measurements. Such a measurement takes approximately $30 \mathrm{sec}$. Each of the images usually contains between 20 and 50 imaged stars. Both images are used for the computation of a single solution of the vertical deflection, cf. Sect. 4.

The DZCS vertical deflection observations analysed in this study were carried out from 2003 to 2006 at a site near the Institut für Erdmessung of the University of Hannover. The observation site is located at the geodetic coordinates

$\varphi=52.386246 \quad \lambda=9.712346$

at an elevation of approximately $50 \mathrm{~m}$ above mean sea level. From 2003 to spring 2004, a minor part of the deflection measurements were eccentrically performed at a near station (distance of about $70 \mathrm{~m}$ ). Due to the completely flat surrounding terrain, similar vertical deflection values are expected at both stations.

From 2003 to 2004, observations at Hannover station were occasionally conducted in order to obtain first estimates of the accuracy of the vertical deflections as observed with a DZCS. Because 
the Hannover DZCS is increasingly involved in gravity field determination projects since 2005, a second aspect motivated regular observations at the same site. The astrogeodetic determination of vertical deflections is an absolute - and not a differential - kind of observation technique, in that, any undetected systematic errors (e.g. due to instrumental drifts or improper modelling of the observations) remain in the data. Therefore, observing vertical deflection data routinely (in our case during approximately three nights per month) is very helpful to monitor the instrumental stability and to detect possible failures of the instrumentation that might reduce the data quality.

The distribution of the complete vertical deflection data set in time is shown in Fig. 1. Over most of the 107 nights 150 or more single observations were performed. Maximum values, ranging between 1000 and 1500 single vertical deflection observations per night, were reached during 5 nights in January 2006. These data already provided important information on the characteristics of anomalous refraction, an error source discussed in Sect. 5.4. It is further seen that the complete observation series falls into two intervals, interrupted by the reworking break in winter 2004/2005. The first interval (identical with the first level of performance) contains the observations conducted over 24 nights in 2003 and 2004. Some results obtained from the analysis of the first 14 of the 24 nights were already reported by Hirt et al. (2004).

The second interval comprises the observational data collected during 84 nights in 2005 and 2006. This interval corresponds to the second level of performance of the DZCS. The dense distribution of these data is additionally shown in Fig. 2. Some gaps of the order of a few weeks are visible which are due to measurement campaigns taking place outside Hannover where the DZCS TZK2-D was involved in. Table 1 gives the detailed observation statistics for each year (rows 1 to 4), for the different levels of performance (rows 5 and 6), as well as for the complete data set (row 7). The table shows that most of the 27300 single observations (about 23600) were acquired since 2005. The total observation time needed for the acquisition of the complete data was about 276 hours and a total of 1.8 million stars were astrometrically processed.

\section{Data processing}

The vertical deflection observations were processed using the software system AURIGA (Automatic Real-Time Image Processing System for Geodetic Astronomy) which is described in detail in Hirt (2001); Hirt and Bürki (2002); Hirt (2004). In brief, the AURIGA data processing chain is as follows. The imaged stars are extracted from the digital zenith images using image moment analysis or, alternatively, a least squares fit with point spread functions. Reference stars are pro- 
vided by the high density UCAC (United States Naval Observatory CCD Astrograph Catalog, Zacharias et al. 2004) or Tycho-2 (Høg et al. 2000) star catalogues which are the state-of-the-art realizations of the International Celestial Reference System ICRS in the optical domain. These catalogues provide reference star coordinates for a couple of million stars accurate to $0.02-0.1$. After star identification and astrometric data reduction, the direction of the principal camera axis is interpolated into the star field, separately for both camera orientations. The mean of both interpolation results is corrected for instrumental tilt and the influence of Earth orientation (sidereal time and polar motion), yielding to the direction of the plumb line $(\Phi, \Lambda)$. Considering the geodetic coordinates $(\varphi, \lambda)$ of the camera (from GPS), vertical deflections $(\xi, \eta)$ are obtained (Eqs. 1 and 2).

\section{Results and accuracy analysis}

A single observation or single solution always refers to vertical deflection values computed from one pair of star images acquired in opposite camera directions. Nightly mean values are obtained by averaging all single solutions obtained during the night. Correspondingly, annual mean values denote the average computed from the nightly mean values collected over one year.

\subsection{Single observations}

Following the processing procedure described in Sect. 4, 3663 single solutions were computed from the data collected in $2003-2004$ and 23616 single solutions for $2005-2006$. The results base on star positions from UCAC, as this catalogue - contrary to Tycho-2 - provided enough reference stars for all regions of the sky appearing in zenith above Hannover over the seasons. The distribution of the $(\xi, \eta)$ values is shown in Fig. 3 and the statistics is compiled in Table 2. It is seen that the observations under level of performance 1 are less accurate (standard deviations of $0^{\prime \prime} .34$ for $\xi$ and 0.31 for $\eta$ ) than those under level of performance 2 (standard deviation of 0 ".22 for both components). The distribution of the 23616 observations from 2005 and 2006 is almost perfectly Gaussian in shape. The visible improvement in accuracy between both levels of performance mainly originates from the mitigation of the azimuth-dependent error of the DZCS due to applying the refined observation technique described in Sect. 2. Moreover, also the efforts made in completely automating the instrumentation are assumed to contribute to the considerable 
data quality of the second level of performance, as any influences caused by the operator are kept away from the data.

\subsection{Nightly and annual mean values}

For each of the 107 nights, the $(\xi, \eta)$ single observations were averaged in order to reduce the observational noise. The resulting $(\xi, \eta)$ nightly mean values are shown in Figs. 4 and 5 as a function of the observation epoch and in Fig. 6 as 2D-plot. The figures clearly show two different levels of data quality which are directly related to the two levels of performance introduced in Sect. 2. For the 23 observations in 2003 and 2004, the standard deviation has been found to be 0 "'12 for the deflection component $\xi$ and 0 " 10 for $\eta$. Since 2005, the noise level of the vertical deflection data is significantly reduced in comparison to the first level of performance. The standard deviation computed from 84 nightly mean values is 0 . 045 both for $\xi$ and $\eta$. This corresponds to an improvement by a factor of 2.5 between both levels of performance. The main contribution to this improvement is made by the mitigation of the azimuth dependent error. A second contributor to the reduced noise level is the somewhat larger average number of single observations available since 2005 for the computation of the mean values. The statistics of the nightly mean values is given in Table 3. The table shows that the East-West vertical deflection component $\eta$, which depends on the time measurement with GPS, is as accurate as $\xi$ (cf. Sect. 5.3).

Table 4 lists the annual as well as the total mean values for the vertical deflections $(\xi, \eta)$ computed from the complete data set. For the $\xi$-observations in 2003 and 2004, somewhat larger annual residuals of about $0{ }^{\prime \prime} 04$ and $0{ }^{\prime \prime} 07$ occur which are attributable to the azimuth error not completely removed from the data collected at level of performance 1. A second reason is related to the weaker data situation in 2004 (a relatively small number of single observations that covers just 1.3 months of the year 2004). Furthermore, it can not be excluded that the eccentric location of the 2003 and 2004 observations has a small influence in terms of the local refractivity as well as in terms of differing expected vertical deflection values. For 2005 and 2006, the annual residuals of the component $\xi$ are $0{ }^{\prime \prime} 022$ and $0{ }^{\prime \prime} 006$, respectively. For the component $\eta$, the annual residuals are generally below 0 !'015.

Fig. 7 focuses on the level of performance 2 data and shows the 84 highly-accurate $(\xi, \eta)$ nightly mean values obtained since 2005 as well as the annual mean values. The spreading width is small with about 0.22 in both components. The data in Table 4 shows that the annual mean values 2005 and 2006 are in agreement of $0{ }^{\prime \prime} 016$ (component $\xi$ ) and $0{ }^{\prime \prime} 009$ (component $\eta$ ). This 
satisfactory agreement originates from the extraordinary large observational redundancy (about 7900 observations collected over 39 nights in 2005 and about 15700 observations over 45 nights in 2006) as well as from the very effective reduction of the instrumental error sources.

\subsection{Formal error estimation}

A formal error estimation is suited for the assessment of the error sources which affect the vertical deflection observations. Error sources with random behaviour are the astrometric processing of the digital star images, the tilt corrections, the registration of the exposure epochs and the determination of geodetic coordinates $(\varphi, \lambda)$ with GPS. The latter plays an insignificant role in the error budget, as differential positioning techniques provide an accuracy level well below 0.01 . The known instrumental error sources with systematic character, such as the zero offsets of the $C C D$ and tilt sensors and the delay of the shutter of the CCD camera, are assumed to play a negligible part in the error budget due to sophisticated measurement and calibration techniques. Since 2005, this also applies for the azimuth-dependent error, as shown in the previous sections. The most important external error caused by the atmosphere is anomalous refraction (cf. Hirt 2006). Depending on the time scale, this effect may show either systematical or random character (Sect. 5.4). A further external error source is a minor systematic error of the UCAC star positions. Below, these error sources are discussed based on investigations by Hirt (2004).

A direction to a single star, as determined in the digital zenith images with image moment analysis or fitting with point spread functions, is usually accurate to 0 "' $3-0$.'4 . These numbers already include the impact of atmospheric scintillation. The positions of reference stars, as provided by the UCAC catalogue, are accurate to $0{ }^{\prime \prime} 02-0{ }^{\prime \prime} 07$, depending on the magnitude (cf. Zacharias et al. 2004). They are therefore of minor significance to the astrometric error budget. On average, about 60 stars are used for the astrometric data reduction and the interpolation of the coordinates of the principal camera axis. The interpolation is usually performed at the accuracy level of 0 "' $15-0$ "'?3. Depending on the amplitudes of scintillation, the interpolation accuracy may exceed 0 "'3 under unfavourable atmospheric conditions.

Reference star positions taken from the current release of the UCAC catalogue are known to have small wave-like systematic errors with amplitudes of up to $0 !$. 015 whereas the Tycho2 catalogue is practically free of systematic errors (cf. Zacharias et al. 2000). The differences between Tycho- 2 and UCAC star positions were confirmed by the vertical deflection observations at Hannover station, which could be processed with both catalogues. Although the systematic 
UCAC catalogue error is of minor significance to the error budget of the vertical deflections, it may reach significance in high-precision astronomical levelling as shown in Hirt and Flury (2007). It is expected that the systematic position errors will be reduced in the forthcoming final UCAC release in 2007.

A GPS receiver is used for registration of the exposure epochs (Sect. 2). The electromechanical shutter of the CCD imaging device, needed for exposure control, is linked to the GPS receiver for time-tagging. Using the electromechanical shutter requires a thorough calibration of its motion characteristics as a function of the temperature. The accuracy of the epoch registration procedure, which is limited by the shutter calibration and not by the GPS timing capability, has been found to be $1 \mathrm{~ms}$. The corresponding error in astronomical longitude $\Lambda$ is $00^{\prime \prime} 015$. As a consequence, homogenous accuracy values are attained both for astronomical latitude $\Phi$ and longitude $\Lambda$, and the deflection components $(\xi, \eta)$, respectively (cf. Sect. 5.2). This uniformity in accuracy is a main benefit of the modern DZCS technology, unlike traditional astrogeodetic techniques.

Tilt measurements formed one of the limiting parts of conventional astrogeodetic instruments (cf. Wissel 1982, Bürki 1989). Significant advancements were made by using new high-resolution tilt meters for the Hannover DZCS. These sensors are provide tilt corrections accurate to 0.!04 0.05 . In order to access this low noise level, the scale factors of the levels are precisely calibrated, an affine model is applied for the computation of tilt corrections and the impact of the zero offsets is eliminated by measurement strategies, as described in Sect. 2 .

The discussed random error sources are compiled in Table 5. As a general conclusion, the error budget of single $(\xi, \eta)$ observations is dominated by the astrometric data processing, particularly by the direction measurements to reference stars.

\subsection{Redundancy of observation}

One of the most important questions related to the application of the new DZCS in field projects is the number of observations required for attaining a certain level of accuracy. This issue is directly related to the efficiency of the DZCS for vertical deflection measurements. The vertical deflection data set collected since 2005 consists of 150 or even more single observations which are available for most of the nights (cf. Fig. 2). Obviously, this redundancy of observation is much higher than normally needed for an accurate determination of vertical deflections. Therefore, the data is suited for a decomposition into several subsets, allowing to investigate the relation between the number of observations and the accuracy achieved. 
In a first step, just one single observation per night is introduced as a nightly mean value. In a second step, the first and second single observation of each night are averaged and represent the nightly mean value. In a next step, the average of the first three single observations of each night is used for the computation of the nightly mean values. Following this approach, a series of nightly mean values simulates the observation of $1,2,3, \ldots, 150$ single observations per night over 84 nights in 2005 and 2006 (level of performance 2 data). In order to smooth the resulting empirical functions of the standard deviations $\sigma_{\xi}$ and $\sigma_{\eta}$, the procedure is repeated with additional shifting of the considered single observations for each night, and the results are eventually averaged.

Fig. 8 shows the standard deviations $\sigma_{\xi}$ and $\sigma_{\eta}$ computed from the simulated series of $(\xi, \eta)$ nightly mean values as a function of the number of observations. The standard deviation $\sigma_{\xi}$ and $\sigma_{\eta}$ for the data consisting of one observation per night is about 0.21 , which is in good agreement with the accuracy of single observations listed in Table $2\left(0^{\prime \prime} \cdot 22\right.$ for both components). It is seen that the noise level of the $(\xi, \eta)$ data strongly decreases with increasing redundancy. A redundancy of about 10 single observations yields to standard deviations of about 0 .' 1 in both components. Increasing the number of observations to $40-50$ lead to $(\xi, \eta)$ values accurate to $0 . \prime 06-0$ " 07 . This corresponds to a gain in accuracy of a factor of about 3.5.

The estimated accuracy level of 0. !06 - 0.'07 for the mean of 40-50 single observations is somewhat better than accuracy estimates obtained from double occupations of stations during field campaigns in Northern Germany and Bavaria where the Hannover DZCS was involved in. During these campaigns, at several stations about 50 single observations were repeatedly collected in two nights. The standard deviation, computed from the differences between the nightly mean values of both nights, was found to be about 0".08 (cf. Hirt and Seeber 2006, Hirt and Flury 2007). A higher noise level of the single observations of these campaigns (standard deviation of about 0.3 ) is considered to be the main cause for the difference.

For the range of 60 to 100 single observations, the observational noise slowly declines to the 0 ".05 accuracy level. Additional observations give slightly better precision numbers of up to $0^{\prime \prime} .045$, which corresponds to the accuracy of the nightly mean values already listed in Table 3 . It is seen that the noise reduction is quite similar for both vertical deflection components.

Fig. 8 additionally displays the theoretical error propagation law for uncorrelated observations: $\sigma_{\bar{x}}=\frac{\sigma_{x}}{\sqrt{n}}$

where $\sigma_{x}$ is the accuracy of a single observation, $n$ is the number of single observations and $\sigma_{\bar{x}}$ is the accuracy of the mean value $\bar{x}$, computed from the $n$ single observations. The error propagation 
law is shown with $\sigma_{x}$ set to 0 "'21, corresponding to the noise level of single $\xi$ and $\eta$ observations, respectively. The comparison between the theoretical error propagation law $\sigma_{\bar{x}}$ and the empirical standard deviations $\sigma_{\xi}$ and $\sigma_{\eta}$ shows generally larger $\sigma_{\xi}$ and $\sigma_{\eta}$ values. These results clearly indicate small positive correlations contained in the data which prevent the observational accuracy from going down with the square root of the observations.

The correlation between the single observations can be quantified applying the error propagation law for correlated observations

$\sigma_{\bar{x}}=\sqrt{\frac{1+(n-1) \rho}{1-\rho}} \frac{\sigma_{x}}{\sqrt{n}}$

(Höpcke 1980, p. 56). It allows us to estimate the accuracy of the mean value $\sigma_{\bar{x}}$ as a function of the accuracy $\sigma_{x}$ of the single observations, the number of single observations $n$ and the correlation coefficient $\rho$. The evaluation of Eq. 5 with $\sigma_{x}$ set to 0 "'21 and different correlation coefficients $\rho$ shows a good agreement between the standard deviations $\sigma_{\xi}, \sigma_{\eta}$ and the theoretical error propagation law for correlation coefficients $\rho=+0.04 \ldots+0.05$ (cf. Fig. 8). The authors are aware of the fact that this simple approach assumes a constant correlation between the observations. A more rigorous correlation analysis would require the determination of the (auto)correlation as a function of the lag between the observations. Such an investigation remains as future task.

It is reasonable to assume anomalous refraction to be the cause for the correlation of about $+0.04 \ldots+0.05$ between the single observations. Anomalous refraction most likely results from tilted atmospheric layers of equal density above the observation site (e.g. Ramsayer 1970, Kovalevsky 1998). This effect, which causes additional angular displacements of observed stars fields, is known to reach amplitudes at the order of $0 . \prime 05-0 . ! 2$ at time scales of some hours (Hirt 2006). Whereas anomalous refraction may behave like a systematic error source at shorter time scales, it becomes more random at longer time scales. With current knowledge, the effect of anomalous refraction cannot be rigorously eliminated from the data. It is concluded that anomalous refraction limits the attainable accuracy of vertical deflections observed over one night to the level of $0 . .04-0 . \prime 05$.

A randomization and further reduction in anomalous reduction, however, seems possible by collecting and averaging vertical deflection data during several different nights. This is indicated in Sect. 5.2, where the agreement of annual mean values of 2005 and 2006 has been found to be below $0{ }^{\prime \prime} 02$. Achieving such a large redundancy of observation, as obtained for these results, however, is very time-consuming. Therefore the redundancy approach for reducing anomalous 
refraction to the level of a few $0 . \prime 01$ is considered to be usually inappropriate for practical field applications.

\section{Discussion and Conclusions}

The accuracy of vertical deflections was analysed by means of a comprehensive data set acquired with the Hannover DZCS, a state-of-the-art astrogeodetic measurement system. The data set consists of about 27300 single observations which were collected over 107 nights from 2003 to 2006 at Hannover station. The statistical analysis of the data reveals an accuracy of single observations of about 0.2 , which is limited by the astrometric direction measurements.

A considerable improvement in accuracy is achieved by averaging a number of single observations. During 20 min observation time about 50 single observations are carried out at field stations. The mean values computed from the 50 repeated observations are accurate to about $00^{\prime \prime} 06-00^{\prime \prime} 07$, whereas accuracy estimates obtained from double occupations of stations during field campaigns are about $0{ }^{\prime \prime} 08$. The difference is assumed to originate from a lower noise level of the single observations at Hannover station.

The accuracy analysis shows that the observational noise is further decreased using up to about 100 repeated observations, requiring approximately 1 hour observation time. The related accuracy of these extended observations has been found to be about $0 . .05$. Additional observations performed over the same night, however, do not significantly improve the accuracy of the vertical deflection data. A comparison with the theoretical error propagation law reveals correlations of about $+0.04 \ldots+0.05$ between the single vertical deflection observations. Anomalous refraction is assumed to cause these correlations which limit the accuracy.

The comparison of the annual mean values 2005 and 2006, which are based on an extraordinary large redundancy of observation, shows a very good agreement below 0.02 for $\xi$ and below 0.01 for $\eta$. This result clearly indicates that influences due to anomalous refraction were effectively mitigated to a large extend. It is certainly a not too optimistic assessment that the annual mean values are accurate to a few 0 " 01 . Therefore, Hannover station is well suited to provide highprecision reference coordinates, required e.g. for any kind of comparisons with other astrogeodetic instrumentation as well as for further monitoring of the instrumental stability of the Hannover DZCS.

The results as presented are considered to be well founded as they base on observations performed over 84 (107) nights, and 1.45 (3.55) years, respectively, if both levels of performance are 
taken into account. The observational data covers all seasons as well as all night times and hence a wide spectrum of external and environmental conditions, such as temperature and pressure, and resulting refraction influences. Moreover, comparisons with independent data sets revealed no significant systematic errors (Hirt et al. 2004, cf. Sect. 1). Therefore, the computed accuracy numbers are assumed to indicate the external accuracy of the Hannover vertical deflection data. However, a rigorous determination of the external accuracy is not possible due to the lack of independent comparison data of higher order accuracy.

It is assumed that the results are representative for field stations with similar refraction characteristics as for Hannover station. The individually attainable accuracy, however, depends on the presence of anomalous refraction and scintillation during the observation.

A comparison with the accuracy level of vertical deflections as attained in the analogue era of geodetic astronomy $\left(0.3-0\right.$ - $\left.0^{\prime \prime}\right)$ shows an improvement of about one order of magnitude. Contributions to this improvement are made not only by the new star catalogues Tycho- 2 and UCAC, the use of CCD technology for star imaging and the redundancy of observation, but also by sophisticated measurement, calibration and processing strategies, keeping the instrumental error sources small. To the knowledge of the authors, this is the first time, that the 0.05 accuracy level for vertical deflections was accessed with a transportable instrumentation.

Acknowledgements The development of the Hannover DZCS as well as its use for the observations analysed in this study was supported by German National Research Foundation DFG from 2001-2006 under contract numbers Se 313/21 and Se 313/22. This support is kindly acknowledged. The authors are grateful to Rene Gudat, Timo Kahlmann, Rene Käker, Tobias Krömer, Eiko Münstedt, Birger Reese and Ilka Rehr for the continous support of the observations. Norbert Zacharias (USNO) is kindly thanked for providing preliminary UCAC star fields enabling this study. The authors thank Beat Bürki, Christopher Jekeli and Bill Kearsley for their helpful comments on the manuscript.

\section{References}

Brockmann E, Becker M, Bürki B, Gurtner W, Haefele P, Hirt C, Marti U, Müller A, Richard P, Schlatter A, Schneider D, Wiget A (2004) Realization of a Swiss Combined Geodetic Network (CH-CGN). EUREF'04 Symposium of the IAG Commission 1 - Reference Frames, Subcommission 1-3a Europe (EUREF), Bratislava, Slovakia

Bürki B (1989) Integrale Schwerefeldbestimmung in der Ivrea-Zone und deren geophysikalische Interpretation. Geodätisch-geophysikalische Arbeiten in der Schweiz, Nr. 40. Schweizerische Geodätische Kommission 
Bürki B, Müller A, Kahle H-G (2004) DIADEM: The New Digital Astronomical Deflection Measuring System for High-precision Measurements of Deflections of the Vertical at ETH Zurich. Electronic Proc. IAG GGSM2004 Meeting in Porto, Portugal. Published also in: CHGeoid 2003, Report 03-33 A (ed. U. Marti et al.), Bundesamt für Landestopographie (swisstopo), Wabern, Schweiz

Heiskanen WA, Moritz H (1967) Physical Geodesy. W.H. Freeman and Company, San Francisco

Hirt C (2001) Automatic Determination of Vertical Deflections in Real-Time by Combining GPS and Digital Zenith Camera for Solving the GPS-Height-Problem. Proceed. 14th International Technical Meeting of The Satellite Division of the Institute of Navigation: 2540-2551, Alexandria, VA

Hirt C (2004) Entwicklung und Erprobung eines digitalen Zenitkamerasystems für die hochpräzise Lotabweichungsbestimmung. Wissenschaftliche Arbeiten der Fachrichtung Geodäsie und Geoinformatik an der Universität Hannover Nr. 253. URL: http://edok01.tib.uni-hannover.de/edoks/e01dh04/393223965.pdf

Hirt C (2006) Monitoring and Analysis of Anomalous Refraction Using a Digital Zenith Camera System. Astron Astrophy 459: 283-290. doi: 10.1051/0004-6361:20065485

Hirt C, Bürki B (2002). The Digital Zenith Camera - A New High-Precision and Economic Astrogeodetic Observation System for Real-Time Measurement of Deflections of the Vertical. Proceed. of the 3rd Meeting of the International Gravity and Geoid Commission of the International Association of Geodesy, Thessaloniki (ed. I. Tziavos): 161-166

Hirt C, Flury J (2007) Astronomical-topographic levelling using high-precision astrogeodetic vertical deflections and digital terrain model data. J Geod. doi: 10.1007/s00190-007-0173

Hirt C, Seeber G (2006) High-Resolution Local Gravity Field Determination at the Sub-Millimeter Level using a Digital Zenith Camera System. Dynamic Planet, Cairns 2005 (ed. P. Tregoning and C. Rizos), IAG Symposia 130: $316-321$

Hirt C, Reese B, Enslin H (2004) On the Accuracy of Vertical Deflection Measurements Using the High-Precision Digital Zenith Camera System TZK2-D. GGSM 2004 IAG International Symposium Porto, Portugal (ed. C. Jekeli et al.), Springer, Heidelberg: 197-201

Hirt C, Denker H, Flury J, Lindau A, Seeber G (2006) Astrogeodetic Validation of Gravimetric Quasigeoid Models in the German Alps - First Results. Accepted Paper presented at 1. Meeting of the International Gravity Field Service, Istanbul

Høg E, Fabricius C, Makarov VV, Urban S, Corbin T, Wycoff G, Bastian U, Schwekendiek P, Wicenec A (2000) The Tycho-2 Catalogue of the 2.5 Million Brightest Stars. Astron Astrophy 355: L27-L30

Höpcke W (1980) Fehlerlehre und Ausgleichsrechnung. W. de Gruyter, Berlin, New York

Kovalevsky J (1998) Environmental Disturbances of Astronomical Observations. Astronomical Society of the Pacific (ASP) Conference Series (ed. S. Isobe and T. Hirayama) 139: 89-96

Marti U (1997) Geoid der Schweiz 1997. Geodätisch-geophysikalische Arbeiten in der Schweiz Nr. 56. Schweizerische Geodätische Kommission

Müller A, Bürki B, Kahle H-G, Hirt C, Marti U (2004) First Results from New High-precision Measurements of Deflections of the Vertical in Switzerland. GGSM 2004 IAG International Symposium Porto, Portugal (ed. C. Jekeli et al.), Springer, Heidelberg: 143-148

Müller A, Bürki B, Limpach P, Kahle H.-G, Grigoriadis VN, Vergos GS, Tziavos IN (2006) Validation of marine geoid models in the North Aegean Sea using satellite altimetry, marine GPS data and astrogeodetic measure- 
ments . Paper presented at 1. Meeting of the International Gravity Field Service, Istanbul

Ramsayer K (1970) Handbuch der Vermessungskunde Band IIa - Geodätische Astronomie. Metzlersche Verlagsbuchhandlung, Stuttgart

Torge W (2001) Geodesy, Third Edition. W. de Gruyter, Berlin, New York

Wissel H (1982) Zur Leistungsfähigkeit von transportablen Zenitkameras bei der Lotabweichungsbestimmung. Wissen. Arb. Fach. Vermessungswesen Univ. Hannover Nr. 107

Zacharias N, Zacharias MI, Urban SE, Høg E (2000) Comparing Tycho-2 astrometry with UCAC1. Astron J 120: $1148-1152$

Zacharias N, Urban SE, Zacharias MI, Wycoff GL, Hall DM, Monet DG, Rafferty TJ (2004) The Second US Naval Observatory CCD Astrograph Catalog (UCAC2). Astron J 127: 3043-3059 
Table 1 Statistics of the vertical deflection measurements at Hannover station

\begin{tabular}{cccccccccc}
\hline $\begin{array}{c}\text { Year/ } \\
\text { period }\end{array}$ & $\begin{array}{c}\text { Number } \\
\text { of nights }\end{array}$ & $\begin{array}{c}\text { First night } \\
\text { [yyyy.yy] }\end{array}$ & $\begin{array}{c}\text { Last night } \\
\text { [yyyy.yy] }\end{array}$ & $\begin{array}{c}\text { Time } \\
\operatorname{span}^{1}[\mathrm{yrs}]\end{array}$ & $\begin{array}{c}\text { Time } \\
\text { span [h] }\end{array}$ & $\begin{array}{c}\text { Single } \\
\text { observations }\end{array}$ & $\begin{array}{c}\text { Processed } \\
\left.\text { stars [10 }{ }^{6}\right]\end{array}$ & $\begin{array}{c}\text { Stars per } \\
\text { solution }\end{array}$ & $\begin{array}{c}\text { Level of perfor- } \\
\text { mance of DZCS }\end{array}$ \\
\hline 2003 & 12 & 2003.15 & 2003.94 & 0.79 & 37.6 & 2870 & 0.238 & 83 & 1 \\
2004 & 11 & 2004.21 & 2004.32 & 0.11 & 13.9 & 793 & 0.029 & 36 & 1 \\
2005 & 39 & 2005.25 & 2005.95 & 0.70 & 83.6 & 7873 & 0.556 & 71 & 2 \\
2006 & 45 & 2006.02 & 2006.70 & 0.68 & 141.0 & 15742 & 0.975 & 62 & 2 \\
\hline $2003-2004$ & 23 & 2003.15 & 2004.32 & 1.17 & 51.5 & 3663 & 0.267 & 73 & 1 \\
$2005-2006$ & 84 & 2005.25 & 2006.70 & 1.45 & 224.6 & 23615 & 1.531 & 65 & 2 \\
\hline $2003-2006$ & 107 & 2003.15 & 2006.70 & 3.55 & 276.1 & 27278 & 1.798 & 66 & 1 and 2 \\
\hline
\end{tabular}

${ }^{1}$ computed as difference between the last and first night of the observation period.

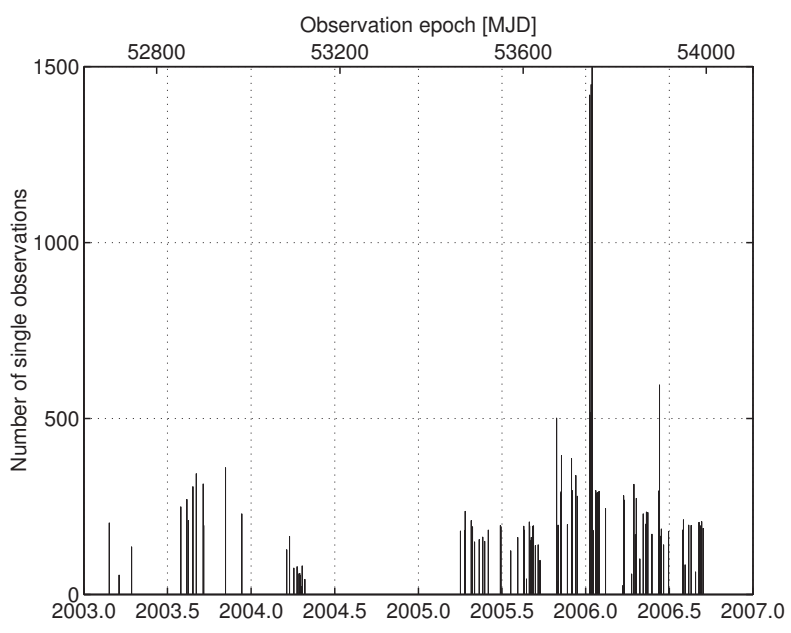

Fig. 1 Number of single observations as a function of the observation epoch (complete data set from 2003 to 2006)

Table 2 Statistics of the single observations

\begin{tabular}{|c|c|c|c|c|c|c|c|c|c|c|}
\hline \multirow{2}{*}{$\begin{array}{c}\text { Period of } \\
\text { observation }\end{array}$} & \multirow{2}{*}{$\begin{array}{c}\text { Level of } \\
\text { performance }\end{array}$} & \multirow{2}{*}{$\begin{array}{l}\text { Number of } \\
\text { observations }\end{array}$} & \multicolumn{4}{|c|}{ Component $\xi$} & \multicolumn{4}{|c|}{ Component $\eta$} \\
\hline & & & $\operatorname{Min}\left[{ }^{\prime \prime}\right]$ & $\operatorname{Max}\left[{ }^{\prime \prime}\right]$ & Mean ["] & Stddev [" $]$ & $\operatorname{Min}\left[{ }^{\prime \prime}\right]$ & $\operatorname{Max}\left[{ }^{\prime \prime}\right]$ & Mean ["] & Stddev ["] \\
\hline 2003-2004 & 1 & 3663 & 4.99 & 8.06 & 6.454 & 0.34 & -0.71 & 2.26 & 1.125 & 0.31 \\
\hline 2005-2006 & 2 & 23615 & 5.00 & 7.40 & 6.516 & 0.22 & -0.23 & 2.01 & 1.109 & 0.22 \\
\hline
\end{tabular}




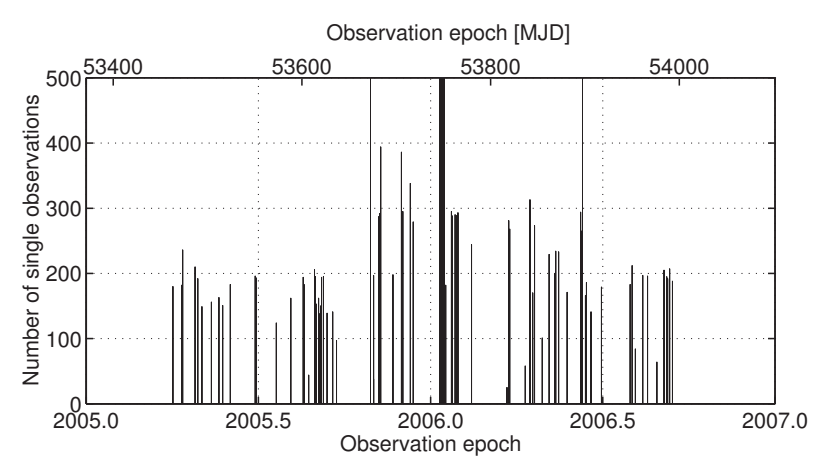

Fig. 2 Number of single observations as a function of the observation epoch (data since 2005, corresponding to level of performance 2)
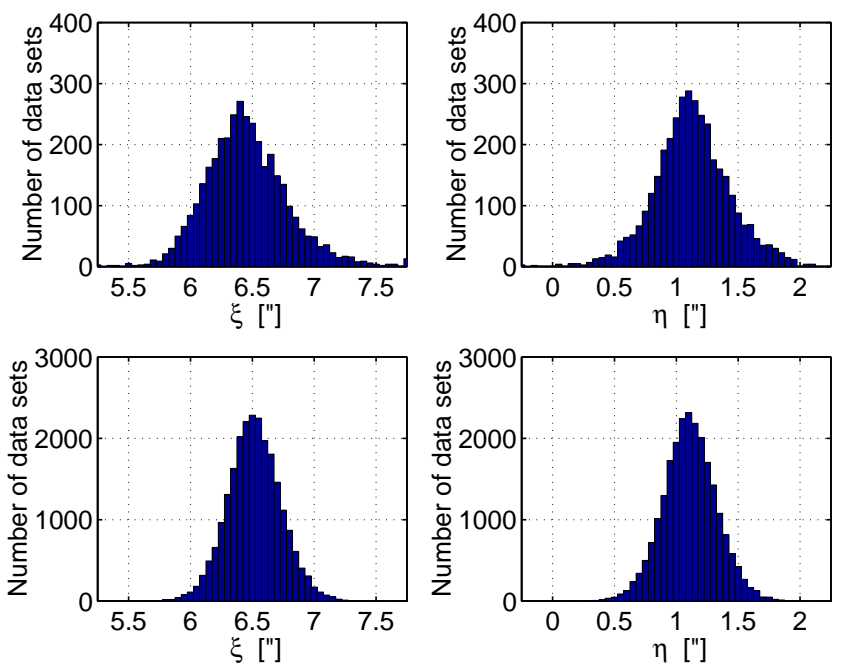

Fig. 3 Above: histogramm of 3663 single $(\xi, \eta)$ observations collected from 2003 to 2004 (level of performance 1). Below: histogramm of 23615 single $(\xi, \eta)$ observations collected from 2005 to 2006 (level of performance 2).

Table 3 Statistics of the nightly mean values

\begin{tabular}{|c|c|c|c|c|c|c|c|c|c|c|}
\hline \multirow{2}{*}{$\begin{array}{c}\text { Period of } \\
\text { observation }\end{array}$} & \multirow{2}{*}{$\begin{array}{c}\text { Level of } \\
\text { performance }\end{array}$} & \multirow{2}{*}{$\begin{array}{c}\text { Number of } \\
\text { nights }\end{array}$} & \multicolumn{4}{|c|}{ Component $\xi$} & \multicolumn{4}{|c|}{ Component $\eta$} \\
\hline & & & $\operatorname{Min}\left[{ }^{\prime \prime}\right]$ & $\operatorname{Max}\left[{ }^{\prime \prime}\right]$ & Mean ["] & Stddev ["] & $\operatorname{Min}\left[^{\prime \prime}\right]$ & $\operatorname{Max}\left[{ }^{\prime \prime}\right]$ & Mean ['] & Stddev $\left[{ }^{\prime \prime}\right]$ \\
\hline 2003-2004 & 1 & 23 & 6.243 & 6.709 & 6.435 & 0.122 & 0.930 & 1.302 & 1.118 & 0.101 \\
\hline 2005-2006 & 2 & 84 & 6.391 & 6.598 & 6.502 & 0.045 & 0.997 & 1.186 & 1.101 & 0.045 \\
\hline
\end{tabular}




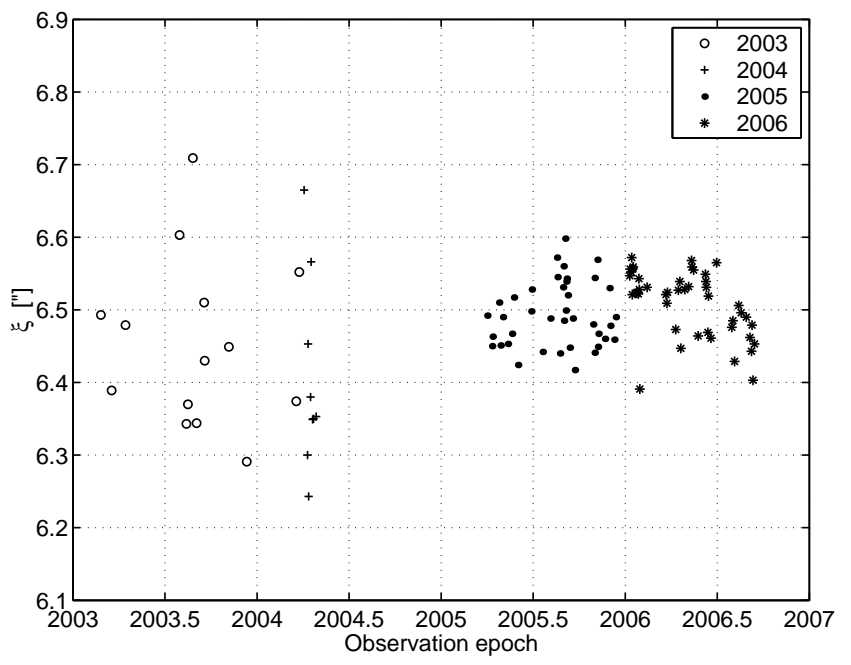

Fig. 4 Nightly mean values of the vertical deflection component $\xi$ as a function of the observation epoch

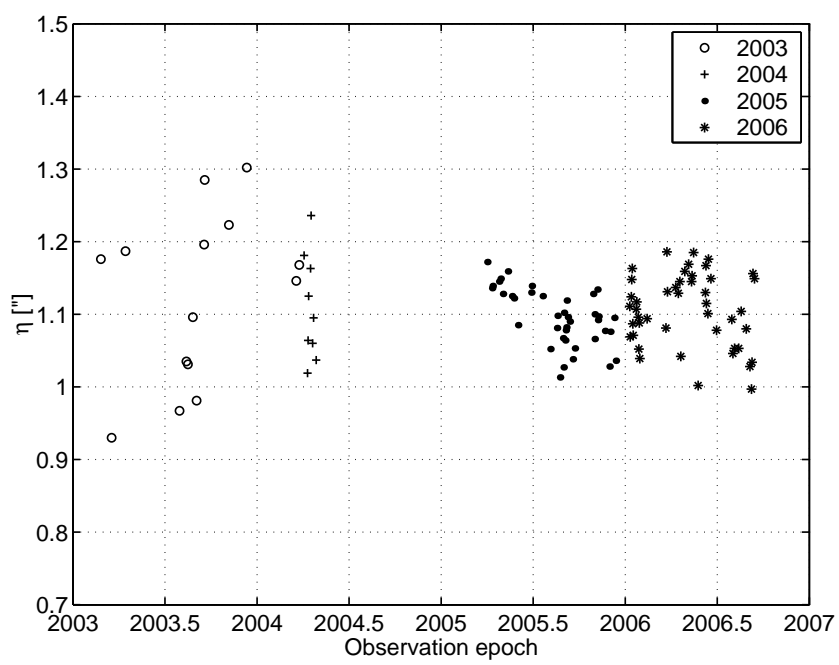

Fig. 5 Nightly mean values of the vertical deflection component $\eta$ as a function of the observation epoch 


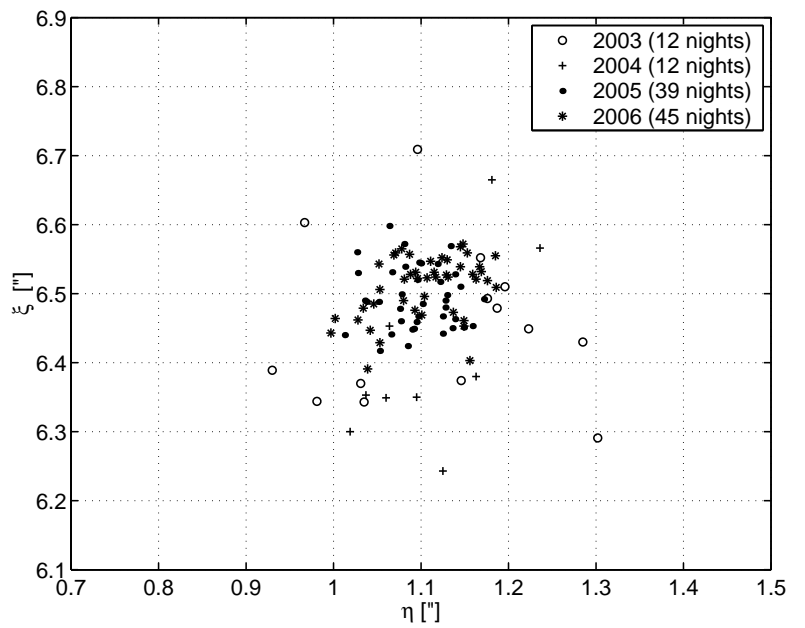

Fig. 6 Nightly mean values $(\xi, \eta)$ (observations of $2003-2006$, levels of performance 1 and 2)

Table 4 Annual results of the vertical deflection measurements and total mean values computed from 27278 single observations from 2003 to 2006 . The annual residuals $r_{\xi}, r_{\eta}$ computed with respect to the total mean values are given in the last columns.

\begin{tabular}{ccrcccc}
\hline Year & Nights & Observations & $\xi\left[^{\prime \prime}\right]$ & $\eta\left[^{\prime \prime}\right]$ & $r_{\xi}\left[^{\prime \prime}\right]$ & $r_{\eta}\left[^{\prime \prime}\right]$ \\
\hline 2003 & 12 & 2870 & 6.451 & 1.117 & 0.037 & -0.013 \\
2004 & 11 & 793 & 6.417 & 1.118 & 0.071 & -0.013 \\
\hline 2005 & 39 & 7873 & 6.493 & 1.096 & -0.006 & 0.009 \\
2006 & 45 & 15742 & 6.509 & 1.105 & -0.022 & 0.000 \\
\hline $2003-2006$ & 107 & 27278 & 6.487 & 1.105 & & \\
\hline
\end{tabular}

Table 5 Formal estimation of the random errors which affect the single observations of vertical deflections

\begin{tabular}{ll}
\hline Error source & Contribution \\
\hline Astrometry (image centering, & $0{ }^{\prime \prime} 15-0{ }^{\prime \prime} 3$ \\
catalogue positions, scintillation) & \\
Tilt corrections & $0{ }^{\prime \prime} 04-0{ }^{\prime \prime} 05$ \\
Time tagging (epoch registration) & $0{ }^{\prime \prime} 015(=1 \mathrm{~ms})$ \\
Geodetic coordinates & $<0{ }^{\prime \prime} 01$ \\
\hline
\end{tabular}




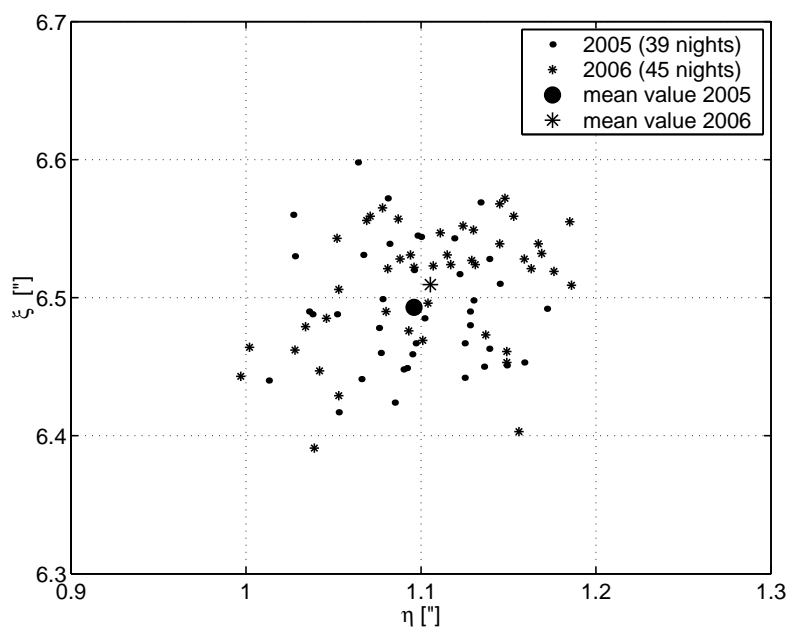

Fig. 7 Nightly mean values $(\xi, \eta)$ (observations of $2005-2006$, level of performance 2)

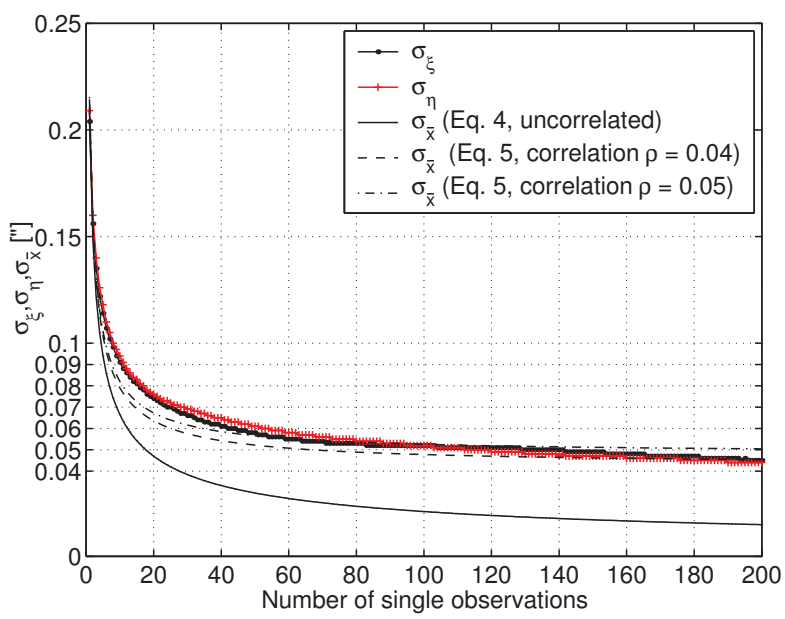

Fig. 8 Accuracy $\sigma_{\xi}$ (bold dotted line) and $\sigma_{\eta}$ (bold line, + marker) as a function of the number of single observations. Additionally, the figure shows the error propagation law for uncorrelated errors (thin solid line) as well as for correlation coefficients $\rho=0.04$ (thin dashed line) and $\rho=0.05$ (thin dashed-dotted line). 